\title{
Correction to: Risk assessments for broncho-pleural fistula and respiratory failure after lung cancer surgery by National Clinical Database Japan
}

\author{
Shunsuke Endo ${ }^{1,2} \cdot$ Norihiko Ikeda $^{1,3} \cdot$ Takashi Kondo $^{1,4} \cdot$ Jun Nakajima $a^{1,5} \cdot$ Haruhiko Kondo $^{1,6} \cdot$ Yoshihisa Shimada $^{1,3}$. \\ Masami Sato $^{1,7}$. Shinichi Toyooka ${ }^{1,8} \cdot$ Yoshinori Okada $^{1,9} \cdot$ Yukio Sato $^{1,10} \cdot$ Ichiro Yoshino ${ }^{1,11}$. Morihito Okada ${ }^{1,12}$. \\ Meinoshin Okumura ${ }^{1,13} \cdot$ Masayuki Chida $^{1,14} \cdot$ Eriko Fukuchi $^{15} \cdot$ Hiroaki Miyata $^{15}$
}

Published online: 5 September 2019

(c) The Japanese Association for Thoracic Surgery 2019

\section{Correction to: \\ General Thoracic and Cardiovascular Surgery \\ (2019) 67:297-305 \\ https://doi.org/10.1007/s11748-018-1022-y}

In the original publication of this article, Tables 1 and 2 were published incorrectly. The correct Tables 1 and 2 are given in this correction.
The original article can be found online at https://doi.org/10.1007/ s11748-018-1022-y.

Shunsuke Endo

tcvshun@jichi.ac.jp

1 Japanese Association for Chest Surgery, 3F Chiyoda Seimei Kyoto Oike Building 200 Takamiya-cho, Takakura-Oike-dori, Nakagyo-ku, 604-0835 Kyoto, Japan

2 Department of Thoracic Surgery, Jichi Medical University, Tochigi, Japan

3 Department of Thoracic Surgery, Tokyo Medical University Hospital, Tokyo, Japan

4 Department of Thoracic Surgery, Tohoku Medical and Pharmaceutical University Hospital, Miyagi, Japan

5 Department of Thoracic Surgery, University of Tokyo Graduate School of Medicine, Tokyo, Japan

6 Department of General Thoracic Surgery, Kyorin University Hospital, Tokyo, Japan

7 Department of General Thoracic Surgery, Graduate School of Medical and Dental Sciences, Kagoshima University, Kagoshima, Japan
8 Department of Thoracic Surgery, Graduate School of Medicine, Dentistry and Pharmaceutical Sciences, Okayama University, Okayama, Japan

9 Department of Thoracic Surgery, Institute of Development, Aging and Cancer, Tohoku University, Miyagi, Japan

10 Faculty of Medicine, Department of Thoracic Surgery, University of Tsukuba, Ibaraki, Japan

11 Department of General Thoracic Surgery, Graduate School of Medicine, Chiba University, Chiba, Japan

12 Department of Surgical Oncology, Hiroshima University, Hiroshima, Japan

13 Department of General Thoracic Surgery, Osaka University Graduate School of Medicine, Osaka, Japan

14 Department of General Thoracic Surgery, Dokkyo Medical University, Tochigi, Japan

15 Department of Healthcare Quality Assessment, Graduate School of Medicine, University of Tokyo, 7-3-1 Hongo, Bunkyo-ku, 113-8655 Tokyo, Japan 
Table 1 Predictors of bronchopleural fistula

\section{$\beta$ coefficient \\ $p$ value \\ Mortality model \\ OR $(95 \%$ CI $)$}

\begin{tabular}{lrrr}
\hline Sex male & 1.014 & $<0.001$ & $2756(1.760-4.315)$ \\
BMI under 18.5 & 0.928 & $<0.001$ & $2.529(1.834-3.488)$ \\
PS2 or over & 0.670 & 0.004 & $1.954(1.231-3.102)$ \\
Interstitial pneumonia & 0.485 & 0.020 & $1.624(1.079-2.446)$ \\
Central nerve system disorder & 0.693 & $<0.001$ & $2.000(1.375-2.910)$ \\
Autoimmune disease & 1.026 & 0.001 & $2.789(1.558-4.995)$ \\
Cigarette smoking habit & 0.659 & 0.007 & $1.934(1.199-3.118)$ \\
Induction radiotherapy or chemoradiotherapy & 0.806 & 0.003 & $2.238(1.309-3.826)$ \\
Clinical N1or higher ${ }^{\mathrm{a}}$ & 0.498 & 0.002 & $1.645(1.202-2.252)$ \\
Clinical stage IB or higher ${ }^{\mathrm{a}}$ & 0.409 & 0.012 & $1.505(1.096-2.068)$ \\
Surgical procedure & & & \\
$\quad$ Right pneumonectomy & 2.460 & $<0.001$ & $11.700(6.518-21.001)$ \\
$\quad$ Right lower lobectomy & 1.412 & $<0.001$ & $4.102(3.137-5.365)$ \\
$\quad$ Left pneumonectomy & 0.897 & 0.026 & $2.451(1.114-5.395)$ \\
$\quad$ Bronchoplasty with segmentectomy or lobectomy & 1.679 & $<0.001$ & $5.358(3.436-8.356)$ \\
$\quad$ Hilar nodal dissection or more & 1.317 & 0.001 & $3.733(1.732-8.047)$ \\
Combined resection & & & \\
$\quad$ Chest wall resection & 0.926 & 0.005 & $2.524(1.315-4.844)$ \\
$\quad$ Wedge resection or segmentectomy of lung & 0.722 & 0.011 & $2.058(1.178-3.596)$ \\
Intercept $(\beta 0)$ & -9.476 & & \\
\hline
\end{tabular}

$B P F$ broncho-pleural fistula, $C I$ confidence interval, $F E V 1$ forced expiratory volume in $1 \mathrm{~s}, P S$ performance status, $V C$ vital capacity

${ }^{\mathrm{a}}$ Clinical staging was clarified by 7 th lung cancer classification 
Table 2 Predictors of respiratory failure

\begin{tabular}{|c|c|c|c|}
\hline & $\beta$ coefficient & $p$ value & $\begin{array}{l}\text { Mortality model } \\
\text { OR }(95 \% \mathrm{CI})\end{array}$ \\
\hline Sex male & 0.467 & 0.004 & $1.595(1.163-2.188)$ \\
\hline Age category ${ }^{\mathrm{a}}$ & 0.219 & $<0.001$ & $1.245(1.156-1.341)$ \\
\hline BMI under18.5 & 0.738 & $<0.001$ & $2.091(1.613-2.710)$ \\
\hline BMI over 30 & 0.682 & 0.008 & $1.978(1.198-3.265)$ \\
\hline Performance status 1 & 0.629 & $<0.001$ & $1.875(1.499-2.345)$ \\
\hline Performance status 2 or higher & 0.724 & $<0.001$ & $2.063(1.452-2.930)$ \\
\hline$\%$ VC $10 \%$ decrease $(100-50 \%)$ & 0.308 & $<0.001$ & $1.361(1.273-1.455)$ \\
\hline FEV1\% 10\% decrease (100-50\%) & 0.550 & $<0.001$ & $1.734(1.343-2.239)$ \\
\hline Liver cirrhosis (Child-Pugh B/C) & 1.323 & $<0.001$ & $3.756(1.807-7.808)$ \\
\hline Interstitial pneumonia & 1.107 & $<0.001$ & $3.024(2.312-3.956)$ \\
\hline Coronary artery disease & 0.422 & 0.006 & $1.524(1.127-2.062)$ \\
\hline Central nerve system disorder & 0.575 & $<0.001$ & $1.776(1.342-2.352)$ \\
\hline Arrhythmia & 0.610 & 0.001 & $1.840(1.301-2.603)$ \\
\hline Cigarette smoking 30 pack-years or more & 0.462 & 0.001 & $1.587(1.214-2.074)$ \\
\hline Tumor size $>3 \mathrm{~cm}$ & 0.343 & 0.005 & $1.410(1.108-1.793)$ \\
\hline Clinical $\mathrm{T} 1 \mathrm{~b}^{\mathrm{b}}$ & 0.299 & 0.025 & $1.349(1.038-1.753)$ \\
\hline Clinical T3 or higher ${ }^{b}$ & 0.350 & 0.033 & $1.419(1.028-1.958)$ \\
\hline Clinical stage III or higher ${ }^{\mathrm{b}}$ & 0.386 & 0.011 & $1.471(1.092-1.980)$ \\
\hline Histology squamous cell carcinoma & 0.369 & 0.001 & $1.447(1.166-1.795)$ \\
\hline Superior sulcus tumor & 0.516 & 0.022 & $1.675(1.079-2.601)$ \\
\hline Multiple lung cancers & 0.984 & $<0.001$ & $2.675(1.722-4.157)$ \\
\hline \multicolumn{4}{|l|}{ Surgical procedure } \\
\hline Right pneumonectomy & 1.538 & $<0.001$ & $4.655(2.507-8.642)$ \\
\hline Right upper lobectomy & 0.350 & 0.002 & $1.418(1.135-1.772)$ \\
\hline Bilobectomy & 1.425 & $<0.001$ & $4.159(2.223-7.782)$ \\
\hline Bronchoplasty with segmentectomy or lobectomy & 0.651 & 0.009 & $1.917(1.174-3.131)$ \\
\hline Hilar nodal dissection or more & 0.813 & $<0.001$ & $2.254(1.591-3.193)$ \\
\hline \multicolumn{4}{|l|}{ Combined resection } \\
\hline Chest wall resection & 0.857 & 0.001 & $2.356(1.447-3.835)$ \\
\hline Intercept $(\beta 0)$ & -9.471 & & \\
\hline
\end{tabular}

$C I$ confidence interval, $F E V 1$ forced expiratory volume in $1 \mathrm{~s}, R F$ respiratory failure, $V C$ vital capacity

${ }^{a}$ The variable of age was categorized into six groups, namely less than 60, 60-64, 65-69, 70-74, 75-79, and more than 80 years old. Therefore, this odds ratio indicates an alteration of relative risk per one unit increase in age category

${ }^{\mathrm{b}}$ Clinical staging was clarified by 7 th lung cancer classification

Publisher's Note Springer Nature remains neutral with regard to jurisdictional claims in published maps and institutional affiliations. 